\title{
Congenital Lung Lesions: Preoperative Three-Dimensional Reconstructed CT Scan as the Definitive Investigation and Surgical Management
}

\author{
${ }^{1}$ Department of Paediatric Surgery, Addenbrooke's Hospital \\ Cambridge, Cambridge, United Kingdom \\ 2 Department of Respiratory and General Paediatrics, Addenbrooke's \\ Hospital Cambridge, Cambridge, United Kingdom \\ ${ }^{3}$ Department of Radiology, Addenbrooke's Hospital Cambridge, \\ Cambridge, United Kingdom \\ Eur J Pediatr Surg 2013;23:53-56.
}

Atif Saeed ${ }^{1}$ Marcin Kazmierski ${ }^{1}$ Abdul Khan ${ }^{1}$ Donna McShane ${ }^{2}$ Anna Gomez ${ }^{3}$ Adil Aslam ${ }^{1}$

\author{
Address for correspondence Adil Aslam, Department of Paediatric \\ Surgery, Addenbrooke's Hospital, Cambridge University Hospitals NHS \\ Trust Foundations, Hills Road, Cambridge CB1 0QQ, United Kingdom \\ (e-mail: adil.aslam@addenbrookes.nhs.uk).
}

\begin{abstract}
Keywords

- lung

- CCAM

- congenital

- cyst

Aim of Study The aim of this study was to review our experience of postnatal investigations and management of congenital lung lesions.

Methods All children with antenatal diagnosis undergoing surgical management were identified from hospital records. Antenatal diagnosis and serial antenatal ultrasound findings were noted, postnatal chest X-ray (CXR) and computed tomographic (CT) scan were reviewed. Pearson correlation coefficient $(r)$ was used to look into relation between $\mathrm{CT}$ scan and per-operative findings. Surgical management and outcome of these lesions were assessed.

Results A total of 38 children were identified between January 2000 and December 2011; 22 were males and 16 were females. The mean gestational age at diagnosis was 21 weeks (range18 to 26 weeks). Five children showed complete resolution antenatally. Four children were symptomatic at birth. Postnatal CXR showed an abnormality in only 17 infants. CT scan with three-dimensional (3D) reconstructions was performed at the mean age of 7.7 months (range 1 day to 42 months). CT scan correlated well with per-operative findings and provided adequate anatomical information $r=0.98$. Open thoracotomy and lobectomy/excision was performed in 23, and 15 had thoracoscopic lobectomy/excision. The mean age of operation was 18 months (range 2 days to 96 months). Twenty patients had signs of recurrent preoperative infection with pleural adhesions and hilar thickening resulting in conversion of 10 thoracoscopic cases to open surgery. Histology confirmed 26 congenital cystic adenomatoid malformations, 2 hybrid lesions, 7 sequestrations, and 3 bronchopulmonary malformations.

Conclusions Antenatal resolution and normal postnatal CXR are not reliable indicators of resolution of the lesion. Early postnatal CT scan preferably with 3D reconstruction and early surgical treatment are suggested, as delaying the operation may result in repeated infection making thoracoscopic approach more difficult.
\end{abstract}

received

May 18, 2012

accepted after revision

January 3, 2013

published online

February 2, 2013 


\section{Introduction}

Congenital lung lesions are rare entities comprising congenital cystic adenomatoid malformation (CCAM), bronchopulmonary sequestrations (BPS), congenital lobar emphysema, ${ }^{1}$ and bronchogenic cysts in majority of cases. Antenatal course of the lung lesions is well studied and it varies from complete resolution to hydrops and fetal death. ${ }^{2}$ However, the ability to predict the postnatal course by antenatal ultrasound (US) is limited. $^{3}$

Postnatal evaluation and treatment of asymptomatic congenital lung lesions are becoming less controversial with most centers opting for the removal of the lesions. ${ }^{4}$ The development of malignancy within CCAM is now increasingly being reported, ${ }^{5}$ hence surgical treatment is suggested by many.

We explored postnatal investigations and management of congenital lung lesions in our center.

\section{Materials and Methods}

A retrospective review of hospital records for surgically managed congenital lung lesions over an 11-year period between January 2000 and December 2011 was done after obtaining approval from the departmental audit and governance committee. Antenatal course was identified from records at the regional fetal medicine department of the Rosie Maternity Hospital, Cambridge University Hospitals NHS Foundation Trust. Antenatal diagnosis was made by US and serial scans were performed to monitor the size and progression of the lesions, which included size of the lesion, assessment of mediastinal shift, amniotic fluid, and hydrops. Postnatal clinical findings and investigation were noted. Chest X-ray (CXR) at birth and computed tomographic (CT) scan with three-dimensional (3D) reconstructions was done at a later date for postnatal evaluation of the lesion. Pearson correlation coefficient $(r)$ was used to look into the relationship between CT scan and per-operative findings. Surgical technique used, operative findings, and postoperative followup were assessed. The patients were reviewed in joint surgical and respiratory clinic. Histological diagnosis were noted and correlated with $\mathrm{CT}$ scan and operative findings.

\section{Results}

A total of 38 children with antenatal diagnosis of the congenital lung lesions were operated during this period; 22 were male and 16 were female. All of them had antenatal anomaly scan at the mean gestational age of 21 weeks (range 18 to 26 weeks).

Five (13.15\%) children showed apparent complete resolution on serial antenatal scans and postnatal CXR; however, postnatal CT scan showed the lesion persisting. Four children were symptomatic immediately after birth. Three required ventilation and one chest intubation for pneumothorax. Seventeen (44.7\%) children developed repeated respiratory symptoms preoperatively. Out of 38 patients, 21 remained asymptomatic. Histological evidence of infection was found in $59 \%$ of asymptomatic children.

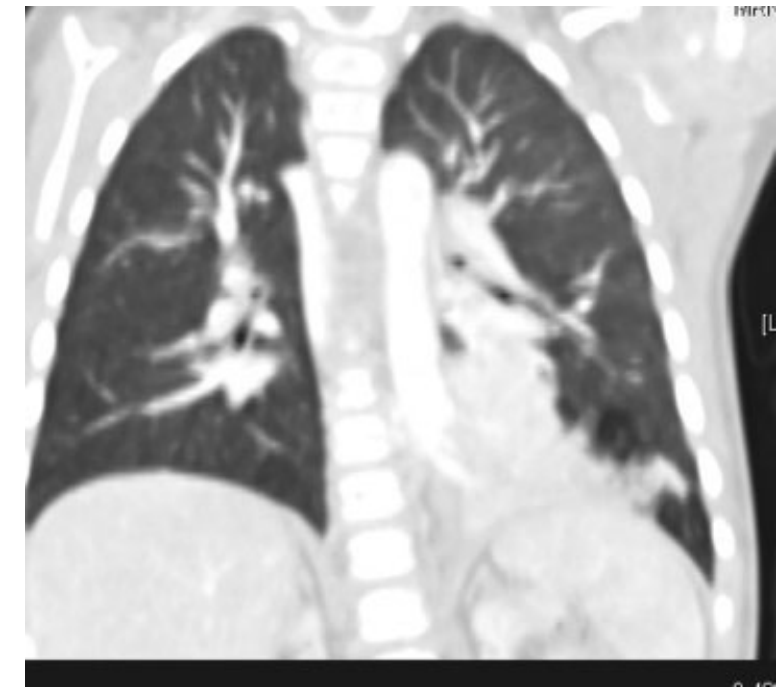

Fig. 1 Computed tomography reconstruction showing systemic vascular supply of bronchopulmonary sequestrations.

Postnatal CXR was unremarkable in 23 antenatal diagnosed patients (60\%). CT scan with $3 \mathrm{D}$ was performed at the mean age of 7.7 months (range 1 day to 42 months). CT scan correlated well with per-operative findings and provided adequate anatomical information $(r=0.98)$, - Figs. 1 and 2 . CT scan showed sensitivity of $87 \%$ and specificity of $100 \%$ in predicting systemic vascular supply for BPS.

The mean age of operation was 18 months (range 2 days to 96 months). Open thoracotomy with lobectomy or excision was performed in 23 patients ( - Table 1 ). In older patients, this involved subperiosteal resection of a rib. Fifteen had thoracoscopic lobectomy or excision ( - Table 1 ). The thoracoscopic operations were done with three $5 \mathrm{~mm}$ ports in the

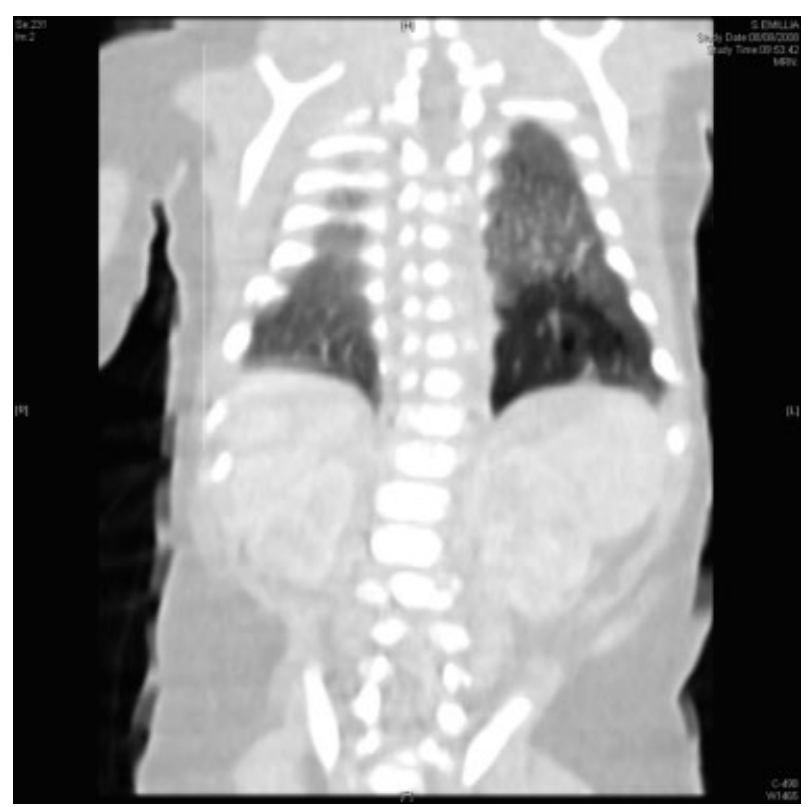

Fig. 2 Computed tomography reconstruction of congenital cystic adenomatoid malformation. 
Table 1 Types of procedures performed

\begin{tabular}{|l|l|l|}
\hline & Thoracoscopic & Thoracotomy \\
\hline Lobectomy & 14 & 20 \\
\hline Excision & 1 & 3 \\
\hline
\end{tabular}

majority of patients. One of the ports was later converted to $12 \mathrm{~mm}$ for introduction of a stapling device and lobe extraction. The hilum was dissected and LigaSure and/or surgical staples were used to seal blood vessels. Twenty children had per-operative signs of recurrent postnatal infection with pleural adhesions and hilar thickening resulting in conversion of 10 thoracoscopic cases to open surgery.

Histology confirmed 26 CCAM, 7 BPS, 2 hybrid lesions, and 3 bronchopulmonary malformations. Chest tube was removed after 24 to 48 hours of the operation. Immediate postoperative complications were delayed chest extubation for small persisting air leak from the chest tube in three patients after thoracotomy and lobectomy. One child developed small port site flail segment after thoracoscopic lobectomy which resolved spontaneously. No difference in complication rate was observed between thoracotomy and thoracoscopic procedure; however, there is significant difference in hospital stay (- Table 2, $p<0.0005)$.

At 3-month follow-up, all the children did well clinically and radiologically. One child had recurrent chest infection, the follow-up bronchoscopy showed bronchomalacia that improved in due course without any surgical intervention.

\section{Discussion}

US has increasingly been used for antenatal diagnosis and monitoring progression of congenital lung lesions. Some of these lesions undergo partial or complete regression. ${ }^{6}$ However, antenatal finding are not predictive of the postnatal course. ${ }^{7}$ Five cystic lesions showed complete resolution on antenatal scans in our study, yet postnatal investigation with CT scan showed the persistence of the lesions. There are also reports of the congenital cystic lesion presented in adults with repeated chest infections. ${ }^{8}$

Postnatal investigations are important and needed to be performed even in the antenatally resolved cases and clinically asymptomatic patients. CXR is the first line of investigations, its accuracy is poor and high false-negative rates were reported. ${ }^{9}$ Sixty percent of patients in our series did not

Table 2 Surgical outcomes

\begin{tabular}{|l|l|l|}
\hline & $\begin{array}{l}\text { Thoracoscopy 25 } \\
\text { Converted 10 }\end{array}$ & $\begin{array}{l}\text { Thorocotomy 23 } \\
\text { Primary 13 }\end{array}$ \\
\hline Complications & 2 & 3 \\
\hline Hospital stay $^{\text {a }}$ (days) & $3 \pm 1.17$ & $5.4 \pm 3.02$ \\
\hline
\end{tabular}

${ }^{\mathrm{a} S}$ Significant difference, $p \leq 0.0005$. show lesion on the postnatal CXR, but it may be useful in showing mediastinal shift or a small pneumothorax. CT scan is the good predictor of the presence and diagnosis of the lesion, and correlates well with surgical and histological findings. In few cases though, it was difficult to differentiate precisely between BPS and CCAM but 3D reconstruction of CT scan images proved useful to illustrate the anatomy better, and guided the surgeon in planning surgery particularly in identifying the systemic vessels supplying BPS. ${ }^{10}$

Treatment of the asymptomatic lesions (monitoring or resection) is becoming less controversial. It has been suggested that nonoperative management for the asymptomatic patients with congenital cystic lesion is appropriate and that only $10 \%$ of the patient developed complications when managed conservatively. ${ }^{11}$ In our opinion, the follow-up of these patients is not only difficult but requires repeated radiation exposure due either to repeated CXR or CT scans. Lifetime cancer mortality risks attributable to radiation from pediatric CT scan examination are estimated to be considerably higher than adult, both because of increased dose per milliampere second and increased lifetime risk per unit dose. ${ }^{12}$

Surgery is recommended on the basis that there is potential risk of the repeated chest infections, pneumothorax, and possible malignancy. Up to 15 cases are reported with associated malignancies both in the children and adults. ${ }^{13}$ The risk of developing pleuropulmonary blastoma is $4 \%$ and mortality rate was described to be $20 \%$ in a recent report. ${ }^{4}$

Initially we inherited a cohort of patients managed conservatively, hence the relatively older mean age of operative interventions. This also may account for our high incidence of initially asymptomatic patients becoming symptomatic later and needing surgical treatment.

We observed that the surgical dissection is difficult to achieve in the cases that were operated when older and they demonstrate either clinical or subclinical infection which were documented on surgical findings and histology. The appropriate time to intervene is not well documented; however, surgical treatment is safe in all age group. ${ }^{14}$ It has also been shown that increasing complications result for delayed and emergency surgery and early surgery at 3 to 6 months of age is recommended, ${ }^{14}$ which we agree with. Better outcomes were reported when surgery is performed in asymptomatic children. ${ }^{15}$ We had to convert 10 thoracoscopic to open lobectomies for the reasons that it was difficult to proceed because of inflammation and adhesions.

\section{Conclusions}

Antenatal resolution on US and normal postnatal CXR are not reliable indicators of resolution of the lesion. Early postnatal CT scan preferably with 3D reconstruction and early surgical treatment are suggested, as delaying the operation may result in repeated infection making the thoracoscopic approach more difficult.

Conflict of Interest

None 
56 Congenital Lung Lesions Saeed et al.

\section{References}

1 Davenport M, Warne SA, Cacciaguerra S, Patel S, Greenough A, Nicolaides K. Current outcome of antenally diagnosed cystic lung disease. J Pediatr Surg 2004;39(4):549-556

2 Illanes S, Hunter A, Evans M, Cusick E, Soothill P. Prenatal diagnosis of echogenic lung: evolution and outcome. Ultrasound Obstet Gynecol 2005;26(2):145-149

3 Williams HJ, Johnson KJ. Imaging of congenital cystic lung lesions. Paediatr Respir Rev 2002;3(2):120-127

4 Nasr A, Himidan S, Pastor AC, Taylor G, Kim PC. Is congenital cystic adenomatoid malformation a premalignant lesion for pleuropulmonary blastoma? J Pediatr Surg 2010;45(6): 1086-1089

5 Oliveira C, Himidan S, Pastor AC, et al. Discriminating preoperative features of pleuropulmonary blastomas (PPB) from congenital cystic adenomatoid malformations (CCAM): a retrospective, agematched study. Eur J Pediatr Surg 2011;21(1):2-7

6 Eber E. Antenatal diagnosis of congenital thoracic malformations: early surgery, late surgery, or no surgery? Semin Respir Crit Care Med 2007;28(3):355-366

7 Sauvat F, Michel JL, Benachi A, Emond S, Revillon Y. Management of asymptomatic neonatal cystic adenomatoid malformations. J Pediatr Surg 2003;38(4):548-552

8 Oh BJ, Lee JS, Kim JS, Lim CM, Koh Y. Congenital cystic adenomatoid malformation of the lung in adults: clinical and CT evaluation of seven patients. Respirology 2006;11(4):496-501
9 van Leeuwen K, Teitelbaum DH, Hirschl RB, et al. Prenatal diagnosis of congenital cystic adenomatoid malformation and its postnatal presentation, surgical indications, and natural history. J Pediatr Surg 1999;34(5):794-798, discussion 798-799

10 Lee EY, Tracy DA, Mahmood SA, Weldon CB, Zurakowski D, Boiselle PM. Preoperative MDCT evaluation of congenital lung anomalies in children: comparison of axial, multiplanar, and 3D images. AJR Am J Roentgenol 2011;196(5):1040-1046

11 Aziz D, Langer JC, Tuuha SE, Ryan G, Ein SH, Kim PC. Perinatally diagnosed asymptomatic congenital cystic adenomatoid malformation: to resect or not? J Pediatr Surg 2004;39(3):329-334, discussion 329-334

12 Brenner D, Elliston C, Hall E, Berdon W. Estimated risks of radiation-induced fatal cancer from pediatric CT. AJR Am J Roentgenol 2001;176(2):289-296

13 Murphy JJ, Blair GK, Fraser GC, et al. Rhabdomyosarcoma arising within congenital pulmonary cysts: report of three cases. J Pediatr Surg 1992;27(10):1364-1367

14 Kim YT, Kim JS, Park JD, Kang CH, Sung SW, Kim JH. Treatment of congenital cystic adenomatoid malformation-does resection in the early postnatal period increase surgical risk? Eur J Cardiothorac Surg 2005;27(4):658-661

15 Marshall KW, Blane CE, Teitelbaum DH, van Leeuwen K. Congenital cystic adenomatoid malformation: impact of prenatal diagnosis and changing strategies in the treatment of the asymptomatic patient. AJR Am J Roentgenol 2000;175(6):1551-1554 